\title{
The cystic fibrosis improvement story: we count our successes in lives
}

\section{Brent C James}

Institute for Health Care Delivery Research, Intermountain Healthcare, Salt Lake City, Utah, USA

\section{Correspondence to Dr Brent C James, Institute for Health Care Delivery Research, Intermountain Healthcare, 36 S. State Street, 16th Floor, Salt Lake City, Utah 84111-1633, USA; brent.james@imail.org}

This editorial refers to the April Supplement, 'Ten years of improvement innovation in cystic fibrosis care,' which publishes alongside this issue of BMJ Quality \& Safety (BMJ Qual Saf 2014;23(Supp/ 1):i1-i108)

Received 14 January 2014 Accepted 15 January 2014 Published Online First 6 February 2014

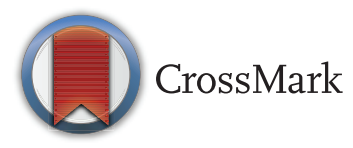

To cite: James BC. BMJ Qual Saf 2014;23:268-271.
It is clear that US health care delivery provides unprecedented health benefits to those who seek its services. It is equally clear that care delivery, and the benefits that it achieves, could be much better. Advances in biomedical research and the application of health care delivery science described in the accompanying supplement have resulted in striking improvements in survival for patients with cystic fibrosis (CF) in the USA. Recent successes in improving CF-related care delivery provide a model to broadly improve health care delivery and patient outcomes on a much wider scale.

\section{CURRENT CARE DELIVERY FALLS SHORT OF ITS THEORETIC POTENTIAL}

About 50 years ago clinical investigators began to apply well-established research measurement tools to health care delivery performance. They discovered massive variation. For example, Wennberg et al documented large differences in hospitalisation rates for comparable patients across geographic referral areas. ${ }^{1}{ }^{2}$ Others showed high levels of practice variation among physicians treating similar patients within single hospitals. ${ }^{3}$ Chassin et al documented high rates of clinically inappropriate care (overuse), where the risk inherent in treatment outweighed any potential clinical benefit to the patient. ${ }^{4}$ A report from the Institute of Medicine conservatively estimated that as many as 98000 hospitalised patients die each year from preventable injuries associated with their treatment (misuse). ${ }^{5}$ Subsequent studies found that the true preventable care-associated death rate is much higher. ${ }^{6}$ McGlynn et al showed that, for a long list of non-controversial, widely-accepted, clearly beneficial clinical interventions based on compelling evidence from randomised controlled trials (RCTs), the care delivery system performs correctly only $54.9 \%$ of the time for adults $^{7}$ and $46 \%$ of the time for children (underuse). ${ }^{8}$

A common theme emerged as investigators tracked possible causes of care delivery variation, care-associated patient injuries, and widespread inability to reliably execute routine care delivery tasks. The healing professions adopted the scientific method as the basis of 'how we know what we know' in the early 1900s. More than 100 years of science, since that time, have greatly improved understanding of the human organism in health and disease, and given clinicians thousands of new treatment interventions. The same explosion in biomedical science also massively increased the complexity of medical science and care delivery operations. Dr David Eddy, the father of evidence-based medicine, said it well: "The complexity of modern medicine exceeds the capacity of the unaided expert mind."9

\section{ADDRESSING THE COMPLEXITY OF MODERN MEDICINE}

Mankind has developed two primary mechanisms to address complexity. The first is the analytic method. When faced with a problem too large to resolve, one could break it down into a series of smaller, more manageable, problems. Solutions created to address each of the small problems then combine into a solution for the large problem. Within the medical profession this 'divide and conquer' approach takes the form of specialisation. A typical medical licence, harking back to a much earlier day, often empowers a physician to 'practice medicine and surgery in all its branches.' In the face of today's vast and rapidly expanding medical knowledge, that assignment is far too broad for any single human mind. A physician might specialise in paediatrics; then, within paediatrics specialise further 
into endocrinology; then, within endocrinology focus even more on diabetes mellitus; and perhaps, even within that much narrowed area, concentrate on CF-related diabetes. Specialisation creates another set of problems. A human patient is far more than the sum of highly specialised, individual subsystems. All of the pieces must somehow come together into a coordinated, comprehensive whole.

The second method of dealing with complexity involves a seeming contradiction: the key to effective, useful variation is standardisation. Start with a standard approach to a common problem, with an embedded measurement and management system to guarantee reliable function every time. Recognising that no two patients are ever exactly the same, a skilled clinician then modifies the standard approach for each individual case. This allows the expert mind to focus on a small subset of the entire problem, where modifications can yield significantly better results. Within quality improvement theory this approach is called 'mass customisation'. ${ }^{10}$ It has been used within clinical practice for generations, but usually within the purview of a single clinician. Today's team-based care demands a broader, more formal application of that proven method.

\section{APPLYING THE RIGOUR OF CLINICAL RESEARCH METHODOLOGY TO CLINICAL PRACTICE}

The same principle of standardisation coupled with focused variation underlies formal clinical research. Clinical trials routinely use protocols to control treatment variation within the arms of a trial. Without such controls it is impossible to tell whether differences in patient outcomes causally arise from the research intervention or from systematic bias introduced by inconsistent treatment.

The method finds an effective application in managing clinical practice variation. In 1991 Morris combined the protocols used to control variation in the arms of RCTs with the tools of quality improvementbased process management. The resulting standard protocols for routine care delivery introduced 'standard work' into a clinical setting while preserving professional autonomy and clinicians' mandate to adjust every treatment plan to the unique needs of an individual patient. ${ }^{11}$ Called Shared Baselines, the method included data systems built into clinical workflows that tracked clinician-initiated protocol variations alongside short- and long-term patient outcomes. A 'learning loop' fed protocol variation and patient outcome data back to clinicians. Clinical teams could not only monitor patient outcomes and care delivery performance, but could systematically validate, update and improve the shared baseline protocols. The resulting data framework made it possible to embed research methods into routine care delivery at a very granular level. In effect every patient became a 'research subject,' contributing reliable knowledge that could improve treatments for future patients, in what has come to be called a Learning Healthcare System. ${ }^{12}$

The method worked. When tried, it dramatically reduced practice variation while improving measured patient outcomes. For example, among the most seriously ill patient sub-cohort in Morris's original adult respiratory distress syndrome (ARDS) trial, survival improved from $<10 \%$ to $44 \% .{ }^{13}$ Lappé et al increased appropriate use of $\beta$-blocker prescriptions at hospital discharge for heart failure patients from $57 \%$ to $98 \%$, which led to a documented decrease in 1-year mortality rates from $22.7 \%$ to $17.8 \%{ }^{14}$ Pronovost et al dropped central line-associated blood stream infection rates from 7.7 to 1.4 infections per thousand line days across 108 intensive care units representing the entire state of Michigan. ${ }^{15}$ Miller et al decreased mortality rates among patients admitted through the emergency department with sepsis from $>20 \%$ to $<8 \%$ in four large tertiary, Utah-based, hospitals. ${ }^{16}$ The list of successful efforts to improve clinical outcomes by addressing variation in clinical practice goes much further than these few illustrations. Equally important, the vast majority of efforts also showed significant reductions in the associated costs of health care delivery operationsvery often, better care is cheaper care.

\section{THE CF IMPROVEMENT STORY}

Perhaps the most striking illustration of reliable care delivery driving massive improvements in clinical outcomes is to be found in the treatment of CF. Between 2002 and 2012, median life expectancy for CF patients in the USA increased from 32 to 41 years. ${ }^{17}$ To my knowledge no other branch of clinical practice in our era can claim such a stunning achievement in lives extended, function maintained, and suffering reduced. We truly do count our successes in lives.

The story of that success is a functional primer on clinical leadership for improved patient outcomes. In 2002 the Cystic Fibrosis Foundation (CFF) hired Dr Bruce Marshall, already skilled in process management methods, to lead their clinical improvement programme. The CFF next invited a group of national clinical quality improvement leaders to a planning meeting in New Hampshire. Two major initiatives arose from that meeting. First, the CFF began to update its shared CF data systems to a level that could support clinical process performance management and embedded clinical learning. The data system update included new policies that expanded data transparency. Second, the CFF sponsored a training programme in clinical improvement methods.

The training programme was launched in November 2003. Intermountain Healthcare in Salt Lake City, Utah, provided a CF-focused specialised version of its Advanced Training Programme in Clinical Practice Improvement (ATP). Teams from CF 
care centres across the USA attended. At the concluding fourth session in January 2004, participating clinical teams presented 12 successful improvement projects that addressed optimal CF nutrition management, the protection and medical management of pulmonary function in CF patients, and management of CF-related diabetes. Following that initial kick-off, Dartmouth's Dr Gerald O'Connor and colleagues continued a series of CFF-sponsored improvement collaboratives. Those programmes provided ongoing hands-on learning coupled with steady, significant improvement in CF care delivery and outcomes.

\section{BIOMEDICAL RESEARCH AND HEALTH CARE DELIVERY SCIENCE}

Each year the US government invests almost $\$ 31$ billion ( $£ 19$ billion, $€ 23$ billion) in biomedical research through the National Institute of Health. ${ }^{18}$ Voluntary health organisations such as the CFF invest additional funds to support research. That investment is producing impressive results. For example, recent trials have identified a pharmaceutical agent, ivacaftor, that corrects the gating defect in the CFTR protein for patients with the G551D mutation in the CFTR gene. ${ }^{19}$ About $4 \%$ of all CF patients who have that particular mutation may achieve very significant longterm health benefits. Other more widely applicable CFTR modulator drugs are in late stage clinical development. Contrast the total impact of that important scientific achievement with what has been obtained by understanding and applying clinical process management science to the care of CF patients, as described in the accompanying supplement. Based on long experience within Intermountain Healthcare, we estimate that the medical profession could achieve as much benefit for patients if it could routinely apply and deliver current best medical knowledge, as will be achieved by the next 25 years of government funding for new biomedical research.

\section{A MASSIVE OPPORTUNITY: HEALTH CARE DELIVERY COULD BE MUCH BETTER}

Obviously the healing professions should vigorously pursue both courses-new biomedical research and care delivery science-at the same time, as has been done in CF. Application of the tools of rigorous clinical research to care delivery performance is a natural extension of well-established science. It holds the potential to greatly accelerate the production of useful clinical evidence, while helping to bring medical evidence to every patient who could benefit from its appropriate use. It represents a massive opportunity for clinical scientists and the patients that we serve.

Competing interests None.
Provenance and peer review Not commissioned; externally peer reviewed.

\section{REFERENCES}

1 Wennberg J, Gittelsohn A. Small area variations in health care delivery: a population-based health information system can guide planning and regulatory decision-making. Science 1973;182:1102-8.

2 Institute of Medicine Committee on Geographic Variation in Health Care Spending and Promotion of High-Value Care.

In: Newhouse JP, Garber AM, Grahma RP, McCoy MA, Mancher M, Kibria A, eds. Variation in health care spending: target decision making, not geography. Washington, DC: National Academy Press (http://www.nap. edu), 2013.

3 James BC. What is a TURP? Controlling variation in the performance of clinical processes. In: Blumenthal DB, Scheck AC, ed. Improving clinical practice: total quality management \& the physician. San Francisco: Jossey-Bass Publishers, 1995: chapter 7.

4 Chassin MR, Galvin RW. The urgent need to improve health care quality-Institute of Medicine National Roundtable on Health Care Quality. JAMA 1998;280:1000-5.

5 Institute of Medicine Committee on Quality of Health Care in America. In: Kohn LT, Corrigan JM, Donaldson MS, eds. To err is human: building a safer health system. Washington, DC: National Academy Press (http://www.nap. edu), 2000.

6 James JT. A new, evidence-based estimate of patient harms associated with hospital care. J Patient Saf 2013;9: 122-8.

7 McGlynn EA, Asch SM, Adams JL, et al. The quality of health care delivered to adults in the United States. New Engl J Med 2003;348:2635-45.

8 Mangione-Smith R, DeCristofaro A, Steodji CM, et al. The quality of ambulatory care delivered to children in the United States. New Engl J Med 2007;357:1515-23.

9 Eddy DM. A manual for assessing health practices \& designing practice policies: the explicit approach. Philadelphia: The American College of Physicians, 1992:14.

10 Womack J, Jones D, Roos D. The machine that changed the world. New York City: Harper Collins, 1991.

11 James BC, Savitz LA. How Intermountain trimmed health care costs through robust quality improvement efforts. Health Affairs 2011;30:1185-91.

12 Institute of Medicine Committee on the Learning Health Care System in America. In: Smith M, Saunders R, Stuckhardt L, Michael MJ, eds. Best care at lower cost: the path to continuously learning health care in America. Washington, DC: National Academy Press, 2013.

13 Morris AH, Wallace CJ, Menlove RL, et al. Randomized clinical trial of pressure-controlled inverse ratio ventilation and extracorporeal $\mathrm{CO}_{2}$ removal for adult respiratory distress syndrome. Am J Respir Crit Care Med 1994;149(2 Pt 1):295-305.

14 Lappé J, Muhlestein JB, Lappé DL, et al. Improvements in 1-year cardiovascular clinical outcomes associated with a hospital-based discharge medication program. Ann Intern Med 2004;141:446-53.

15 Pronovost P, Needham D, Berenholtz S, et al. N Engl J Med 2006;355:2725-32. 
16 Miller RR 3rd, Dong L, Nelson NC, et al. Multicenter implementation of a severe sepsis and septic shock treatment bundle. Am J Respir Crit Care Med 2013;188:77-82.

17 Marshall BC, Nelson EC. Accelerating implementation of biomedical discovery: critical elements of a successful 10-year cystic fibrosis healthcare improvement initiative. BMJ Qual Saf 2014; in press.
18 National Institutes of Health. NIH budget. http://www.nih.gov/ about/budget.htm (accessed 8 Jan 2014).

19 Ramsey BW, Davies J, McElvaney NG, et al. A CFTR potentiator in patients with cystic fibrosis and the G551D mutation. N Engl J Med 2011;365:1663-72. 